\title{
ABSTRACTS OF THESES
}

J.R. Trollope, Exact Solutions of the Einstein-Maxwell Equations that Determine Null Fields, University of Alberta, Edmonton.

The role of the null electromagnetic field, within the framework of classical relativistic electrodynamics, has never been fully established. The object of this dissertation has been to present a large class of solutions to the Einstein-Maxwell null field equations. It has been found that the nature of the solutions depend on the form of a fundamental null vector. In the cases for which the divergence of this vector vanishes, an extensive class of solutions has been obtained. The alternate case, when the divergence is non-zero, has not been fully investigated. In the latter case, it has been possible to obtain a particular solution only.

J.C. Ahuja, Statistical Problems in the Study of Growth, University of British Columbia, June 1963. (Supervisor: S. W. Nash).

The problem of estimating the parameters of several growth curves has been considered for the case where repeated correlated observations are taken on the same individual or population. The se curves are the logistic, the Gompertz, the modified exponential, the $\theta$-generalized logistic, and their modified forms with lower asymptotes different from zero. Three methods of estimation have been suggested and the mathematical procedure of each has been discussed.

The different methods of estimation yield the vector equations for the estimators whose solutions require the inverse of the variance and covariance matrix. A procedure is given for obtaining the inverse of the type of covariance matrix used in our model. The procedure given holds good for all matrices of this type of any order and does not require the use of computers.

The methods of estimation suggested are all of iterative type and require starting values of the parameters. A method for obtaining the starting values of the parameters has been given for each curve. The method for obtaining the starting values involves the estimation of the derivatives of the growth function $w(t)$ or $\log w(t)$ with respect to $t$. The differentiation formulas for the estimation of the se derivatives from the observed data, when the series of values may be given at equal or unequal intervals, have been obtained.

The stochastic models for the logistic, the Gompertz, and the 
modified exponential laws of growth have been formulated as pure birth Markov processes. The solutions of the differential-difference equations describing the probability laws of the processes have been obtained by solving the partial differential equations for their generating functions. The properties of the processes have been studied by deriving the expressions for the means, variances and correlations. A method for obtaining the maximum likelihood estimators of the parameters involved has also been given in each case.

The problem of distinguishing the different phases of growth has been attacked by deriving orthogonal expansions from the logistic, the Gompertz, and the exponential densities, in a manner similar to the way in which Gram (1879) and Charlier (1906) derived an orthogonal expansion from the normal density.

The $\varphi$-generalized Gompertz, the $(\varphi, \theta)$-generalized logistic, and the $(\varphi, \theta)$-generalized modified exponential densities have been obtained as generalizations of the Gompertz, the $\theta$-generalized logistic, and the $\theta$-generalized modified exponential respectively. The limiting cases of these densities have been found as $\varphi$ or $\theta$ or both are allowed to go to infinity or zero.

Lastly, the recurrence relation for the orthogonal polynomials $q_{n}(x)$ (leading coefficient one) of degree $n$ associated with the density function $f(x)$ over the interval $[a, b]$ has been derived explicitly in terms of the moments of $f(x)$. Further, an alternative proof has been given of the theorem that if $f(x)$ is symmetrical about $x=0$, then the polynomials $q_{n}(x)$ are even or odd functions according as $n$ is even or odd. 